\title{
Molecular Models of Wolbachia-Induced Cytoplasmic Incompatibility Must Account for the Multiplicity of Compatibility Types
}

\author{
Alice Namias ${ }^{1}$, Mathieu Sicard ${ }^{1}$, Mylène Weill ${ }^{1, *}$, Sylvain Charlat ${ }^{2, *}$
}

1. ISEM, University of Montpellier, CNRS, IRD, Montpellier, France

2. Laboratoire de Biométrie et Biologie Evolutive, Université de Lyon, Université Lyon 1, CNRS, UMR 5558, 43 boulevard du 11 novembre 1918, Villeurbanne, F-69622, France

* corresponding authors : sylvain.charlat@univ-lyon1.fr ; mylene.weill@umontpellier.fr

\begin{abstract}
Wolbachia endosymbionts commonly induce cytoplasmic incompatibility, making infected males' sperm lethal to the embryos unless these are rescued by the same bacterium, inherited from their mother. Causal genes were recently identified but two families of mechanistic models are still opposed. In the toxin-antidote model, interaction between the toxin and the antidote is required for rescuing the embryos. In host modification models, a host factor is misregulated in sperm and rescue occurs through compensation or withdrawal of this modification. While these models have been thoroughly discussed, the multiplicity of compatibility types, i.e., the existence of many mutually incompatible strains, as seen in Culex mosquitoes, has not received sufficient attention. To explain such a fact, host modification models must posit that the same embryonic defects can be induced and rescued through a large variety of host targets. Conversely, the toxin-antidote model simply accommodates this pattern through variations in the toxin-antidote interaction sites.
\end{abstract}

Keywords: Wolbachia, toxin-antidote, symbiosis, Culex, Drosophila 


\section{1 - Wolbachia and cytoplasmic incompatibility in arthropods}

Cytoplasmic incompatibility $(\mathrm{Cl})$ denotes a phenomenon of conditional sterility induced by various endosymbiotic bacteria, of which Wolbachia is the most renowned. In its simplest form (Fig. 1), Cl occurs when males carrying Wolbachia mate with uninfected females. In such crosses, fertilization takes place normally but most or all embryos die before hatching (1-3). In contrast, development proceeds if the female is infected, regardless of the male's infection status. This means that Wolbachia makes infected females more fertile than infected ones on average and on the contrary renders infected males less fertile than uninfected ones. However, the male side does not matter as far as Wolbachia is concerned since only females transmit the infection to their offspring, through the egg cytoplasm. The infected lineage frequency thus tends to increase, and more effectively so if the infection has no negative side effect and is perfectly transmitted across generations (4). In this perspective, $\mathrm{Cl}$ is understood as a selfish adaptive feature of the symbiont, increasing its chances of invading new species following rare events of horizontal transmission (5).
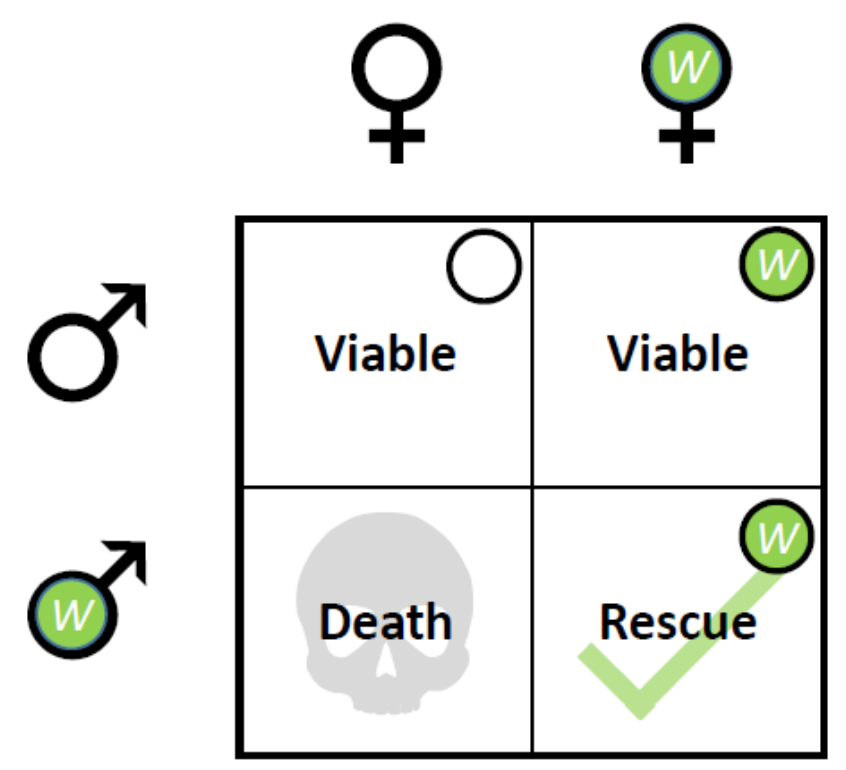

Figure 1: $\mathrm{Cl}$ in its simplest form: compatibility relationships between infected and uninfected individuals. Uninfected females engender fewer viable offspring than infected ones, because they 
are not immune to the sperm produced by infected males. The upper-right circle shows the infection state of the progeny: empty (uninfected) or filled (infected). "W" stands for Wolbachia infection.

Cytological studies indicate that $\mathrm{Cl}$ induces similar cellular phenotypes in a wide range of hosts (6-11). During the first embryonic division, paternal chromosomes exhibit defects in condensation and improperly segregate during anaphase. The resulting embryos are aneuploid or haploid and thus inviable, except in haplodiploid species where they may survive as males (12). While these developmental failures were described a while ago, the underlying genetics have remained elusive until recent years. Because embryonic viability is impeded by the sperm of infected males and rescued by the presence of Wolbachia in the eggs, it has long been formalized that $\mathrm{Cl}$ is a two-sided phenomenon, implying some kind of "modification" in mature sperm and some kind of "rescue" taking place in the eggs $(13,14)$. This modification/rescue or mod/resc model is generally acknowledged as a flexible framework that could accommodate any underlying molecular mechanism.

Two causal genes were recently identified concomitantly in the Wolbachia genomes of major $\mathrm{Cl}$ model systems: the wPip strain from Culex pipiens mosquitoes and the wMel strain from Drosophila melanogaster flies $(15,16)$. In both study systems, these genes, generally referred to as "Cl factors" (cif) form an operon-like structure in the prophage WO region (16). Transgenic expression of the upstream gene (cifA) is necessary and sufficient to prevent $\mathrm{Cl}$ onset in fly embryos, that is, to recapitulate the resc function. Transgenic expression of cifB is toxic in yeasts and this effect is rescued by its coexpression with cifA, making CifB a good "mod factor" candidate. Yet only when cifA and cifB are coexpressed in male flies is $\mathrm{Cl}$ induction effectively recapitulated. Intriguingly, transgenic expression of cifB from another 
strain (wRec, naturally present in Drosophila recens) is sufficient to induce strong $\mathrm{Cl}$-like embryonic mortality, but this effect is not rescuable, casting doubt on the possibility that it caused the same molecular defects as dual cifA/B expression (17). Putting aside this particular case, transgenic expression of the CifA protein thus seems paradoxically required for both $\mathrm{Cl}$ rescue and induction. This may indicate that CifA displays two distinct and somewhat opposite molecular activities depending on the context, making it a mod co-factor in maturing sperm and a resc factor in embryos, as proposed in the so-called "two-by-one model" (18). Alternatively CifA may just act as a rescue factor, required not only for the normal development of embryos but also for preventing the inherent toxicity of CifB during spermatogenesis (19). Within the cif genes family, at least two active operons have been identified, that can be specifically referred to as cid and cin, based on the enzymatic activity, deubiquitinase or nuclease, of their downstream protein. Yet, additional homologs have been described and the cif genes are generally distributed into five clades (16, 20-22). Thus far, $\mathrm{Cl}$ induction has been experimentally associated with cid and cin operons, respectively falling into clades I and IV, and also with one operon from group II, but putative functional domains have been identified in all five groups $(15,17,18,23)$.

Now that the key $\mathrm{Cl}$ effectors have been identified, understanding their molecular mechanism will require deciphering what these proteins actually do. Yet, the scope of possibilities remains very large, making it relevant to evaluate the various plausible mechanistic models in light of the current data. Several recent review papers thoroughly discussed these models $(19,24-27)$ but perhaps did not put enough emphasis on one specific question that may help guiding future research: based on current knowledge, should we expect the $\mathrm{Cl}$ rescue to stem from a direct interaction between CifA and CifB in the 
embryos? Answering this question will essentially distinguish two families of mechanistic models that are still opposed, namely host-modification (HM) versus toxin-antidote (TA) models (Fig. 2) $(17,19,27,28)$.

As recently summarized (e.g. $(17,19))$, under TA models, the mod and resc factors act like a lock and key: the toxin (mod) affecting the paternal chromosomes is transported through sperm into the embryo where its interaction with the antidote (resc) is needed to prevent the modification and thereby let the first mitosis proceed (Fig. 2A). On the contrary, HM models propose that the mod/resc interaction is only indirect and happens through host effectors: the mod factor(s) induces paternal DNA defects but is not transferred into the embryo so that rescue happens by reversing its effects (Fig. 2B). In fact, in one particular HM version, called the "mistiming model", the mod and resc sides of $\mathrm{Cl}$ stem from a single process: slowing down the mitotic dynamics; under this model, embryos are only viable if the paternal and maternal pronuclei are synchronous. A second, perhaps more abstract HM version, called the "goalkeeper model", assumes modification takes place in males in a strain-specific quantity, needing to be remedied to rescue embryos. Finally, the "titrationrestitution" model posits that Wolbachia alters the concentration of a crucial host effector in sperm and thus induces $\mathrm{Cl}$ unless this is counter-balanced in the embryo. 


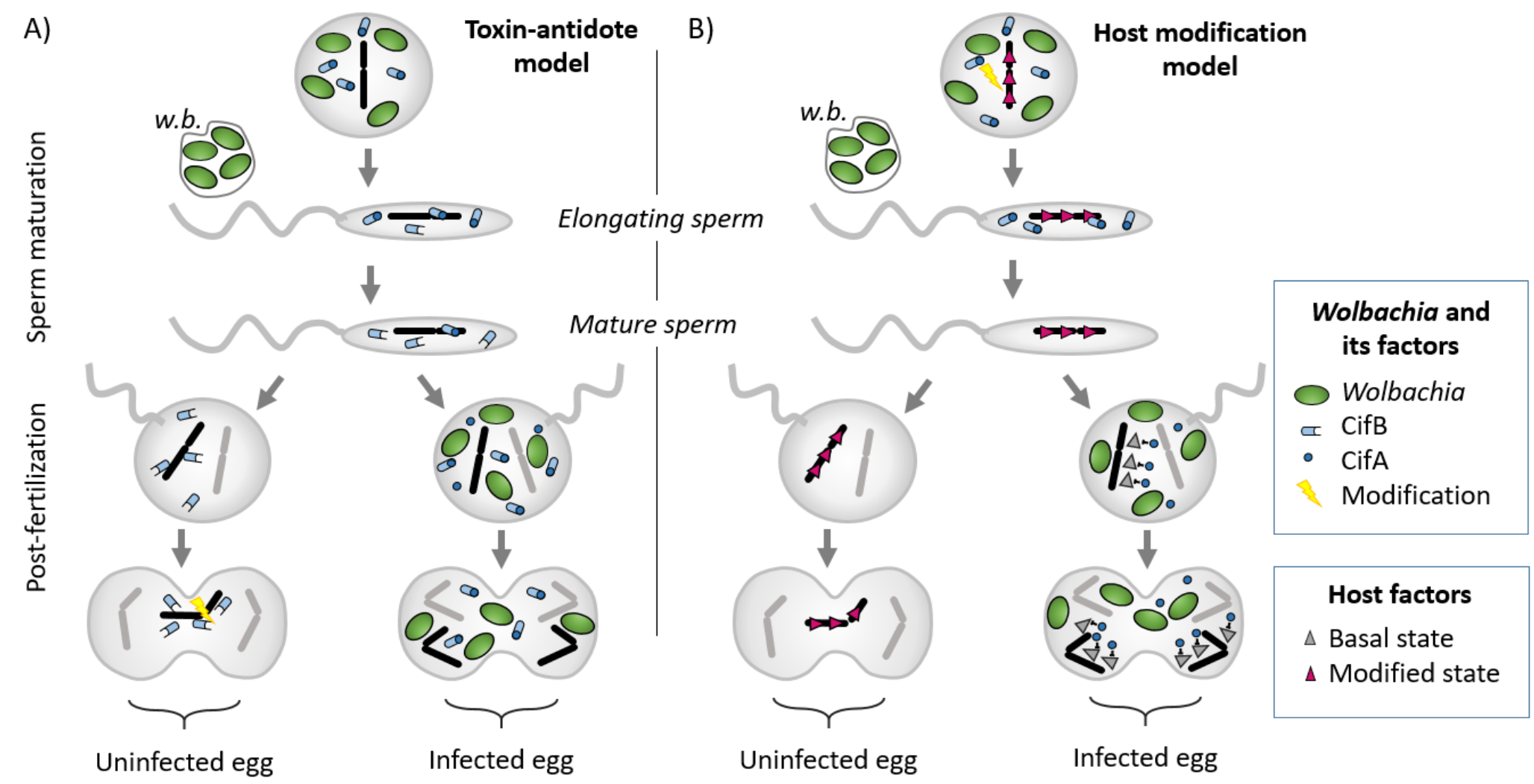

Figure 2: Schematic views of the two main families of $\mathrm{Cl}$ mechanistic models. In both frameworks,

Wolbachia is removed from maturing sperm into waste bags (w.b.). a) The toxin-antidote (TA) model predicts that a toxin produced by the paternal Wolbachia is transported with paternal DNA into the eggs and causes mitotic defects unless it is directly inhibited by an antidote produced by the maternal Wolbachia. The paternal antidote may also be required during sperm development but is presumably less stable than the toxin and would thus be degraded prior to fertilization. b) In Host Modification (HM) models, a host factor is modified in the sperm. This modification causes developmental defects unless CifA in the egg inhibits/reverses its effects. In both frameworks, the maternal CifA proteins would constitute the rescue factor, while paternal CifB, with a debated contribution of paternal CifA, would be responsible for $\mathrm{Cl}$ induction.

Which of these two families of models is most likely correct? Binding assays provide some elements of answer by showing that the CidA and CidB proteins, as well as CinA and CinB, tightly bind in vitro, while CidA only binds to few other elements ((29), resp. (23)). While not 
ruling out HM models, this pattern indicates that a central requirement of TA models is likely fulfilled although it still awaits in vivo confirmation. Notably, another central requirement of the TA model, namely, the production of the CidB protein in sperm and its conveyance into the eggs has not been documented yet, neither in Culex nor in Drosophila. However, such absence of evidence should not be taken as evidence of absence, because this protein has not been specifically searched for in these tissues. In any case, we now turn to consider a different line of arguments, building on the multiplicity of Wolbachia compatibility types.

\section{2 - Compatibility types: a simple case study}

We have so far described $\mathrm{Cl}$ in its simplest form, that occurring between infected males and uninfected females, a pattern often referred to as unidirectional $\mathrm{Cl}$, because the reverse cross produces viable progeny (Fig. 1). Yet it has long been known that $\mathrm{Cl}$ may also occur between males and females that are both infected, but by distinct Wolbachia strains (Fig. 3). The Drosophila simulans system provides a rather simple situation to apprehend this phenomenon (reviewed in (30)). This species naturally hosts several Wolbachia lineages, usually called "strains", easily distinguished on the basis of standard molecular markers such as $16 \mathrm{~S}$ rRNA. Some of these strains happen to have lost the ability to induce $\mathrm{Cl}(31,32)$ but three of them are still capable of both $\mathrm{Cl}$ induction and rescue: $w \mathrm{Ri}, w \mathrm{Ha}$, and $w \mathrm{No}$, originally described in lines from Riverside (California), Hawaii and Noumea (New Caledonia), respectively. These three strains display quantitative differences with regard to $\mathrm{Cl}$ penetrance but most importantly, they are all bidirectionally incompatible. In that sense, they are said to harbor distinct "compatibility types". 
A)
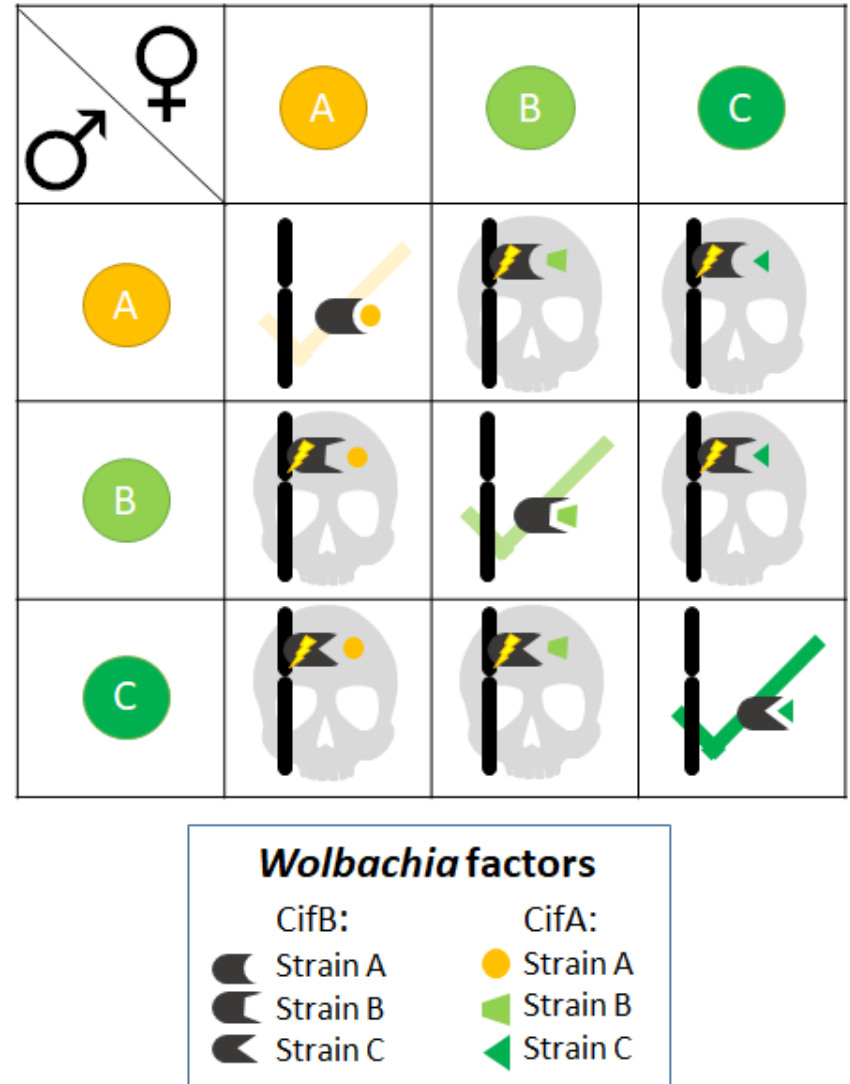

B)

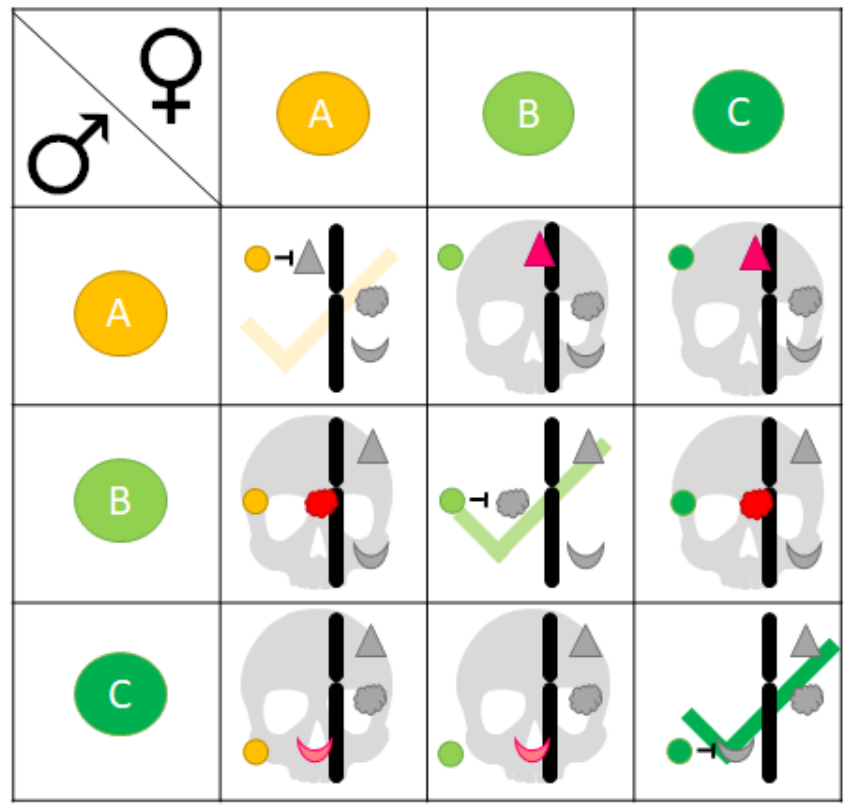

Wolbachia factors

CifA:

- Strain A

Strain B

- Strain C

\section{Host factors}

Basal state Modified state $\triangle$ Strain A Strain A

Strain B

Strain C
Strain A
Strain B Strain C

Figure 3: A simple case of mutual incompatibilities explained under the TA and HM frameworks. a)

In TA models, compatibility patterns can be explained by a lock-and-key analogy: rescue occurs only

if the antidote (the key) can appropriately bind the toxin (the lock), preventing or reversing

modifications of the paternal chromatin. Bidirectionally incompatible Wolbachia strains carry

incompatible locks and keys (as represented here by non-complementary shapes). Various toxins and

antidotes would thus differ in their protein-protein interaction regions, but not necessarily in their

functional domains (represented here with a constant shape). b) In the HM framework, mutually

incompatible Wolbachia produce mod factors that necessarily target different host effectors, and are

specifically matched by their resc counterpart. These various pathways would lead to the same

paternal chromatin defects.

To what extent can the TA and HM frameworks account for such mutual incompatibilities

(Fig. 3)? Within the TA model, the multiplicity of compatibility types is readily explained by

the possibility that distinct mod/resc pairs could differ, not in their targets, but in their 
interaction sites, as the Lock-Key analogy makes obvious (Fig. 3A). Within the HM family, the mistiming model, while elegant in proposing that $\mathrm{Cl}$ induction and rescue may stem from a single phenomenon, just fails to explain the existence of more than one compatibility type, as previously noted (28). In contrast, other HM models may account for mutual incompatibilities, but this would imply that the mod factor(s) of incompatible Wolbachia strains achieve the same cytological defects through the activation of different host effectors (Fig. 3B). Furthermore, the resc factors should similarly vary in their targets to specifically inhibit the consequences of their mod counterpart (Fig. 3B). The above-mentioned existence of more than one functional family within $\operatorname{cifB}$ genes $(\operatorname{cid} B, \operatorname{cin} B$ and possibly other yet uncharacterized enzymes) could form the basis of such a diversity. Transgenic expressions in yeast provide indirect evidence that the cid and cin operons from wPip may be mutually incompatible: CidA and CinA can only rescue the toxicity respectively caused by CidB and CinB (15). Yet the same approach suggested that compatibility types do not simply come down to enzymatic families: while the toxicity associated with cidB from wHa was fully rescued by its cognate cidA, expression of the cidA gene from wPip only lead to imperfect rescue (29). The existence of incompatibilities within functional families is further suggested by pull down assays indicating that CidB from wPip only interacts with its cognate CidA, and not with either CinA from wPip or CidA from the wMel operon (15). Recent transgenesis experiments in Drosophila provide mixed results regarding the degree of specificity associated with different Cif factors from distinct strains carrying clade I and/or clade II operons, presumably differing in their enzymatic activities (17). The $\mathrm{Cl}$ induced by two distinct clade I operons was interchangeably rescued by their CifA factors, suggesting compatibility would be preserved within the deubiquitinase functional family, at least between the two Wolbachia strains tested. Yet, surprisingly, a clade I CifA protein was found 
able to rescue the $\mathrm{Cl}$ induced by a clade II operon, although the reciprocal was not true. Coming back to the aforementioned Wolbachia strains from $D$. simulans, it is noticeable that the wRi genome carries both a type I (cid) and a type II operon, while wHa harbors only a type I operon and $w$ No only a type III operon $(16,20)$. Assuming compatibility is preserved within functional families, embryos carrying the $w$ Ri strain should be immune to the $\mathrm{Cl}$ induced by $w \mathrm{Ha}$. Yet they are not, which further highlights that incompatibility patterns cannot be readily reduced to enzymatic families.

In brief, the TA model can rather simply explain bidirectional incompatibilities through changes in the CifA-CifB interaction sites. While this model does not rule out the possibility that different Wolbachia strains may also achieve similar $\mathrm{Cl}$ through different effectors (as suggested by the fact that both nuclease and deubiquitinase activities may produce similar cytological phenotypes) but it does not rely on this enzymatic diversity to explain mutual incompatibilities (Fig. 3A). The HM framework also remains compatible with the existence of more than one compatibility type. Mutual incompatibilities would then stem from differences in the host targets of the distinct mod factor(s), appropriately matched by their resc counterparts (Fig. 3B). The existence of more than one functional family offers at best a partial explanation for observed compatibility patterns. While still plausible, the HM framework then faces the challenge of explaining that a variety of distinct pathways would lead to the same cytological phenotype. As we shall now discuss, a further challenge is to explain that compatibility types are substantially more diverse than suggested by the Drosophila case. 


\section{3 - Compatibility types: a bigger picture}

While the $D$. simulans case is sufficient to demonstrate that different Wolbachia strains may be mutually incompatible, the scale of this phenomenon is better apprehended by considering data from Culex pipiens. Even before Wolbachia was identified as the causal agent, this species was known for displaying complex patterns of incompatibilities among distinct geographic populations (33-35). Following the observation that, in contrast with $D$. simulans, C. pipiens only hosts one Wolbachia "strain" (named wPip) as far as standard molecular markers such as $16 \mathrm{~S}$ rRNA can tell, it has been hypothesized that host genetic variation likely contributed to this complexity (36). Yet, more discriminant molecular markers soon revealed that the $w$ Pip clade is in fact composed of several lineages (37) and more importantly, experiments controlling for the host genetic background repeatedly indicated that Wolbachia alone is responsible for the observed incompatibilities $(34,35,38)$.

Once acknowledged, this feature provides important insights with regard to Wolbachia compatibility types. First, they are numerous: the number of mutually incompatible strains in $C$. pipiens exceeds by far those reported in $D$. simulans. For instance, by reciprocally crossing 19 different $C$. pipiens lines, 15 distinct compatibility profiles have been revealed $(34,39)$. Second, they can diverge very rapidly, as indicated by the study of intra-population $\mathrm{Cl}$ variations (40-43). Third, they can be asymmetrical: strain $\mathrm{A}$ may rescue the $\mathrm{Cl}$ induced by strain B without the reciprocal being true. Fourth, they can be non-transitive: strains A and B may be mutually compatible and yet display distinct compatibility patterns with strain C (35, 44). On the basis of these properties, it was possible to predict not only that the $\mathrm{Cl}$ genes should display an important diversity in C. pipiens, but also that a single Wolbachia genome should carry more than one mod/resc pair $(35,39)$. 
The recently uncovered diversity of the cif genes in wPip matched these predictions rather neatly (42). Both cin and cid genes are found in wPip genomes but all strains carry these two enzymatic types of $\mathrm{Cl}$ effectors, ruling out the hypothesis that incompatibilities could result from carrying either one or the other. Most critically, while the cin genes are monomorphic in all wPip genomes studied so far, the cid genes are amplified and diversified, with up to six different copies of a given cid gene within a single genome (42). Furthermore, cid diversity strongly correlates with $\mathrm{Cl}$ patterns $(42,43)$. Notably, mutual incompatibilities have nothing to do with differences in the Cid deubiquitinase functional domain, which is monomorphic, but rather with polymorphism in putative CidA/CidB interaction sites $(42,43)$. It may be obvious already to the reader that these latter results better fit a TA than an HM framework. The large diversity of compatibility types constitutes a major difficulty for the HM models, because it unreasonably multiplies the number of distinct host targets, both on the males and females sides, through which Wolbachia strains would produce the same cellular phenotype. Much more parsimoniously, within the TA framework, mutual incompatibilities can simply result from differences in CidA/CidB interaction sites. Intermediate affinities among Cid proteins and quantitative variations in their production could also readily explain the intermediate levels of rescue recently described (38).

It is arguably premature to conclude that the HM versus TA debate is over. A better understanding of what the Cif proteins actually do will be required to reach that point. However, unless one assumes that the Culex case represents an oddity relying on peculiar mechanisms, the multiplicity of Wolbachia compatibility types weighs heavy in the balance and has perhaps received too limited attention in previous discussions of $\mathrm{Cl}$ mechanistic models $(17,19,27)$. Among other approaches, further studies focusing on the binding affinities between different versions of the wPip Cid proteins along with a deeper 
assessment of cid genes diversity in this system will certainly contribute to deciphering the mechanisms and evolution of $\mathrm{Cl}$.

\section{References}

1. Marshall JF, Staley J. 1937. Some notes regarding the morphological and biological differentiation of Culex pipiens Linnaeus and Culex molestus Forskal (Diptera, Culicidae). Proc R Entomol Soc London 12:17-26.

2. Yen JH, Barr AR. 1971. New hypothesis of the cause of cytoplasmic incompatibility in Culex pipiens L. Nature 232:657-658.

3. Yen JH, Barr AR. 1973. The etiological agent of cytoplasmic incompatibility in Culex pipiens. J Invertebr Pathol 22:242-250.

4. Turelli M, Hoffmann AA, McKechnie SW. 1992. Dynamics of cytoplasmic incompatibility and mtDNA variation in natural Drosophila simulans populations. Genetics 132:713-723.

5. Sanaei E, Charlat S, Engelstädter J. 2020. Wolbachia host shifts: routes, mechanisms, constraints and evolutionary consequences. Biol Rev 96:433-453.

6. Ryan SL, Saul GB. 1968. Post-fertilization effect of incompatibility factors in Mormoniella. Mol Gen Genet MGG 103:29-36.

7. Lassy CW, Karr TL. 1996. Cytological analysis of fertilization and early embryonic development in incompatible crosses of Drosophila simulans. Mech Dev 57:47-58.

8. Callaini G, Riparbelli MG, Giordano R, Dallai R. 1996. Mitotic defects associated with cytoplasmic incompatibility in Drosophila simulans. J Invertebr Pathol 67:55-64.

9. Tram U, Sullivan W. 2002. Role of delayed nuclear envelope breakdown and mitosis in Wolbachia-induced cytoplasmic incompatibility. Science (80- ) 296:1124-1126. 
10. Landmann F, Orsi GA, Loppin B, Sullivan W. 2009. Wolbachia-mediated cytoplasmic incompatibility is associated with impaired histone deposition in the male pronucleus. PLoS Pathog 5:1-9.

11. Bonneau M, Landmann F, Labbé $P$, Justy F, Weill M, Sicard M. 2018. The cellular phenotype of cytoplasmic incompatibility in Culex pipiens in the light of cidB diversity. PLoS Pathog 14:e1007364.

12. Bordenstein SR, Uy JJ, Werren JH. 2003. Host genotype determines cytoplasmic incompatibility type in the haplodiploid genus Nasonia. Genetics 164:223-233.

13. Hurst LD. 1991. The evolution of cytoplasmic incompatibility or when spite can be successful. J Theor Biol 148:269-277.

14. Werren JH. 1997. Wolbachia and speciationEndless forms: Species and Speciaton. Oxford University Press.

15. Beckmann JF, Ronau JA, Hochstrasser M. 2017. A Wolbachia deubiquitylating enzyme induces cytoplasmic incompatibility. Nat Microbiol 2:1-7.

16. LePage DP, Metcalf JA, Bordenstein SR, On J, Perlmutter JI, Shropshire JD, Layton EM, Funkhouser-Jones LJ, Beckmann JF, Bordenstein SR. 2017. Prophage WO genes recapitulate and enhance Wolbachia-induced cytoplasmic incompatibility. Nature 543:243-247.

17. Shropshire JD, Rosenberg R, Bordenstein SR. 2021. The impacts of cytoplasmic incompatibility factor (cifA and cifB) genetic variation on phenotypes. Genetics 217.

18. Shropshire JD, Bordenstein SR. 2019. Two-By-One model of cytoplasmic incompatibility: Synthetic recapitulation by transgenic expression of cifA and cifB in Drosophila. PLoS Genet 15:1-20.

19. Beckmann JF, Bonneau $M$, Chen $H$, Hochstrasser $M$, Poinsot $D$, Merçot $H$, Weill $M$, 
Sicard M, Charlat S. 2019. The Toxin-Antidote Model of Cytoplasmic Incompatibility: Genetics and Evolutionary Implications. Trends Genet 35:175-185.

20. Lindsey ARI, Rice DW, Bordenstein SR, Brooks AW, Bordenstein SR, Newton ILG. 2018. Evolutionary Genetics of Cytoplasmic Incompatibility Genes cifA and cifB in Prophage WO of Wolbachia. Genome Biol Evol 10:434-451.

21. Martinez J, Klasson L, Welch JJ, Jiggins FM. 2021. Life and Death of Selfish Genes: Comparative Genomics Reveals the Dynamic Evolution of Cytoplasmic Incompatibility. Mol Biol Evol 38:2-15.

22. Bing XL, Zhao DS, Sun JT, Zhang KJ, Hong XY, Sloan D. 2020. Genomic Analysis of Wolbachia from Laodelphax striatellus (Delphacidae, Hemiptera) Reveals Insights into Its "jekyll and Hyde" Mode of Infection Pattern. Genome Biol Evol 12:3818-3831.

23. Chen H, Ronau JA, Beckmann JF, Hochstrasser M. 2019. A Wolbachia nuclease and its binding partner provide a distinct mechanism for cytoplasmic incompatibility. Proc Natl Acad Sci U S A 116:22314-22321.

24. Shropshire JD, Leigh B, Bordenstein SR, Duplouy A, Riegler M, Brownlie JC, Bordenstein SR. 2019. Models and Nomenclature for Cytoplasmic Incompatibility: Caution over Premature Conclusions - A Response to Beckmann et al. Trends Genet 35:397-399.

25. Beckmann JF, Bonneau M, Chen $H$, Hochstrasser M, Poinsot D, Merçot $H$, Weill M, Sicard M, Charlat S. 2019. Caution Does Not Preclude Predictive and Testable Models of Cytoplasmic Incompatibility: A Reply to Shropshire et al. Trends Genet 35:399-400.

26. Chen H, Zhang M, Hochstrasser M. 2020. The Biochemistry of Cytoplasmic Incompatibility Caused by Endosymbiotic Bacteria. Genes (Basel) 11:1-21.

27. Shropshire JD, Leigh B, Bordenstein SR. 2020. Symbiont-mediated cytoplasmic 
incompatibility: What have we learned in 50 years? Elife 9:1-36.

28. Poinsot D, Charlat S, Merçot H. 2003. On the mechanism of Wolbachia-induced cytoplasmic incompatibility: Confronting the models with the facts. BioEssays 25:259265.

29. Beckmann JF, Sharma GD, Mendez L, Chen H, Hochstrasser M. 2019. The Wolbachia cytoplasmic incompatibility enzyme CidB targets nuclear import and protaminehistone exchange factors. Elife 8:e50026.

30. Merçot H, Charlat S. 2004. Wolbachia infections in Drosophila melanogaster and D. simulans: Polymorphism and levels of cytoplasmic incompatibility. Genetica 120:5159.

31. Hoffmann AA, Clancy D, Duncan J. 1996. Naturally-occuring Wolbachia infection in Drosophila simulans that does not cause cytoplasmic incompatibility. Heredity (Edinb) 76:1-8.

32. Rousset F, Solignac M. 1995. Evolution of single and double Wolbachia symbioses during speciation in the Drosophila simulans complex. Proc Natl Acad Sci 92:63896393.

33. Laven H. 1967. Eradication of Culex pipiens fatigans through Cytoplasmic Incompatibility. Nature 216:383-384.

34. Duron O, Bernard C, Unal S, Berthomieu A, Berticat C, Weill M. 2006. Tracking factors modulating cytoplasmic incompatibilities in the mosquito Culex pipiens. Mol Ecol 15:3061-3071.

35. Atyame CM, Duron O, Tortosa P, Pasteur N, Fort P, Weill M. 2011. Multiple Wolbachia determinants control the evolution of cytoplasmic incompatibilities in Culex pipiens mosquito populations. Mol Ecol 20:286-298. 
36. Rousset F, Raymond M, Kjellberg F. 1991. Cytoplasmic incompatibilities in the mosquito Culex pipiens: How to explain a cytotype polymorphism? J Evol Biol 4:69-81.

37. Duron O, Lagnel J, Raymond M, Bourtzis K, Fort P, Weill M. 2005. Transposable element polymorphism of Wolbachia in the mosquito Culex pipiens: evidence of genetic diversity, superinfection and recombination. Mol Ecol 14:1561-1573.

38. Sicard M, Namias A, Perriat-Sanguinet M, Carron E, Unal S, Altinli M, Landmann F, Weill M. 2021. Cytoplasmic incompatibility variations in relation with Wolbachia cid genes divergence in Culex pipiens. MBio 12:e02797-20.

39. Nor I, Engelstädter J, Duron O, Reuter M, Sagot MF, Charlat S. 2013. On the genetic architecture of cytoplasmic incompatibility: Inference from phenotypic data. Am Nat 182.

40. Duron O, Bernard J, Atyame CM, Dumas E, Weill M. 2012. Rapid evolution of Wolbachia incompatibility types. Proc R Soc B Biol Sci 279:4473-4480.

41. Atyame CM, Labbé P, Rousset F, Beji M, Makoundou P, Duron O, Dumas E, Pasteur N, Bouattour A, Fort P, Weill M. 2015. Stable coexistence of incompatible Wolbachia along a narrow contact zone in mosquito field populations. Mol Ecol 24:508-521.

42. Bonneau M, Atyame CM, Beji M, Justy F, Cohen-Gonsaud M, Sicard M, Weill M. 2018. Culex pipiens crossing type diversity is governed by an amplified and polymorphic operon of Wolbachia. Nat Commun 9:319.

43. Bonneau M, Caputo B, Ligier A, Caparros R, Unal S, Perriat-Sanguinet M, Arnoldi D, Sicard M, Weill M. 2019. Variation in Wolbachia cidB gene, but not cidA, is associated with cytoplasmic incompatibility mod phenotype diversity in Culex pipiens. Mol Ecol 28:4725-4736.

44. Atyame CM, Labbé $\mathrm{P}$, Dumas E, Milesi $\mathrm{P}$, Charlat S, Fort $\mathrm{P}$, Weill M. 2014. Wolbachia 
divergence and the evolution of cytoplasmic incompatibility in Culex pipiens. PLoS One 9:e87336. 